\title{
An experimental analysis on the performance of single mode-multimode- single mode and multimode- single mode-multimode fiber optic sensor
}

\author{
Nur Hidayah Sulaiman, Hanim Abdul Razak, Hazura Haroon, Anis Suhaila Mohd Zain \\ Centre for Telecommunication Research \& Innovation (CeTRI), Fakulti Kejuruteraan Elektronik dan Kejuruteraan Komputer (FKEKK), \\ Universiti Teknikal Malaysia Melaka (UTeM), Hang Tuah Jaya, 76100, Durian Tunggal, Melaka, Malaysia
}

* Corresponding author: hanim@utem.edu.my

\section{Article history}

Submitted 25 January 2018

Revised 29 March 2018

Accepted 11 July 2018

Published Online 4 February 2019

\begin{abstract}
A cost effective and simple fabrication process for Mach Zehnder Interferometer (MZI) fiber based sensor has been proposed based on single mode-multimode-single mode structure and multimodesingle mode-multimode. These proposed structures employed a standard fusion arc splicing by varying the lengths of sensing region instead of the structures. This sensor has been experimentally demonstrated for three different liquid solutions such as water, $1 \mathrm{~mol}$ sucrose solution and oil with the refractive index of $1.333,1.384$ and 1.464 respectively. Furthermore, the intention of this experiment was to determine which structure that would provide superior performance in terms of the sensitivity of the device. The operating wavelength of different structures was corresponded to the different refractive index. It was observed that the shifting response was influenced by the length of the sensing-area and the best sensitivity achieved was $-10.45 \mathrm{~nm} / \mathrm{RIU}$.
\end{abstract}

Keywords: Fiber optic sensor, interferometer, Mach-Zehnder interferometer (MZI), single modemultimode-single mode (SMS), multimode-single mode-multimode (MSM)

C 2019 Penerbit UTM Press. All rights reserved

\section{INTRODUCTION}

An optical sensors possess the superiorities of low power consumption, immunity and electromagnetic interference, smart structure and high sensitivity [1] compared to conventional sensing techniques such as electrical or mechanical sensors. Fiber optic sensors that can perform a high sensitivity and resolution have been subjected to broad investigations for the measurement of various physical and chemical parameters such as temperature [2], [3], strain [4], [5] humidity [6], [7] and refractive index (RI) [8]-[11].

RI measurement in small volumes becomes an essentials role in many areas of biophysics, biochemistry and biomedicine. It can be used to determine the concentration of sugar or proteins [12]. Biophotonic sensors are optical devices based on optical phenomena to measure biological species such as cells, proteins, and DNA [12], [13]. Since it is very beneficent in terms of efficiency, accuracy, cost and very convenience, the bio-photonic sensors are promising alternatives to traditional immunological methods for biomolecule measurements [12].

In recent years, optical sensors based on micro-structured fiber have been proposed and demonstrated as in surface plasmon resonance [8], [14] and photonic crystals fiber [15], [16]. However by using thin film of gold $(\mathrm{Au})$, silver $(\mathrm{Ag})$ or copper $(\mathrm{Cu})$ for the surface plasmon resonance surface has resulted in limitations that include lacking of uniformity and chemically unstable [14]. In addition, RI measurements can be shown through the fiber Bragg gratings [3-6] and long period gratings [7-9] with very high sensitivity, yet it can still exhibit the cross sensitivity between refractive index and temperature.

On the other hand, interferometers fiber based sensor has also been documented as RI measurements with less complex configuration of fabrication and represented by four types of fiber optic interferometers, called the Fabry-Perot (FPI), Michelson (MI), Sagnac (SI) and Mach-Zehnder (MZI) [17]. The fabrication of the extrinsic FPI is relatively simple, but it executes low coupling efficiency because of the parallel separated mirrors are required to form a cavity and partially reflect the lead-in optical signals as in [18]-[20]. Meanwhile MI has been recorded in [21]-[23] with low sensitivity as the fiber optic sensor. As for SI, researchers reported the drawbacks on the sensitivity of the polarization mirror based on studies by [24], [25]. On the other hand, MZI possesses superior property such that the configuration of fabrication can be in a small size. Most of researchers agreed that the fabrication of the MZI structure is easy to handle for the RI measurement in [10], [26]-[31].

In this article, we proposed two types of MZI structures which were single mode-multimode-single mode (SMS) and multimodesingle mode-multimode (MSM) as fiber optic sensor responding to the RI measurement. To the best of the author's knowledge, this is the first report on the comparison of sensitivity for both SMS and MSM fiber optic sensor on the same platform. The length of sensing region has been varied with $4 \mathrm{~cm}$ and $8 \mathrm{~cm}$ to analyze the effect of different lengths on the performance of the device. These proposed structures have been tested in three different liquid concentrations such as water, 1 mol sucrose solutions and oil that have different values of RI. Moreover, the proposed MZI structures could provide the advantages in terms of cost and fabrication procedures because the fabrication involved the standard fusion.

\section{THEORETICAL BACKGROUND}

The schematic diagram of MZI was given in

Figure 1. It is consisted of two independent arms which are reference arm and sensing arm. An optical signal from the source is passed through into the reference arm. The power of optical signal has been divided perfectly $50 / 50$ by the $3-\mathrm{dB}$ coupler at the splitter while another 3-dB coupler is coupled back the optical signal from both 
arms. The phase optical signal is found to be affected at the sensing arms and produced the interference as entering the receiver [17].

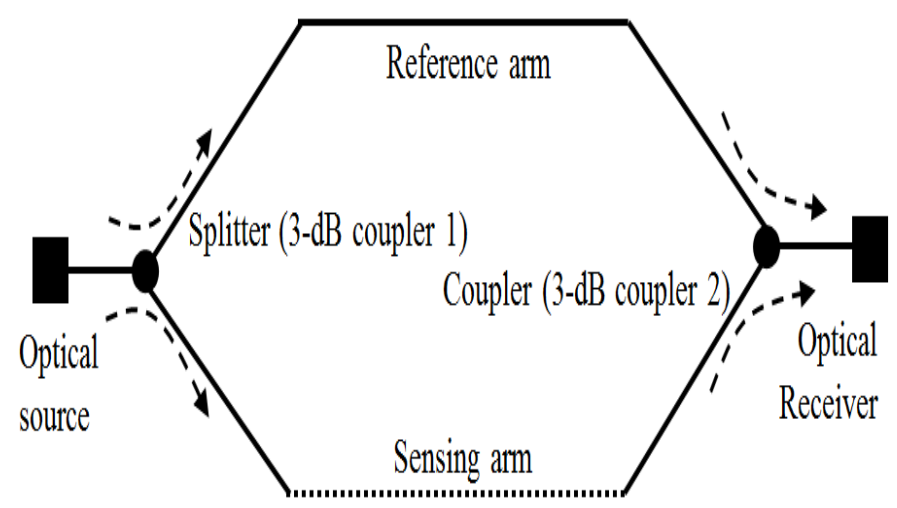

Figure 1 Schematic diagram of Mach Zehnder interferometer.

Generally, lead-in, sensing area and lead-out region are the basic block diagram for these MZI structures as shown

Figure 1. The optical signal is passed into the sensing area region through the lead-in region and that is affected by the mode field diameter mismatch. At the sensing area region, the cladding mode of fiber is represented as the sensing arm while the core mode is represented as the reference arm, which are formed the MZI structure. Furthermore, the sensing area is the region where the sensor is responded to the ambient response. In the second joint, the cladding mode will interfere with the core mode and then couple into leadout region fiber to be received by the detector. The mode field of both fibers' diameter is mismatched and caused the interference at both splicing point.

Figure 2 shows the block diagram of SMS structure involving the single mode fiber (SMF) as the lead-in region and the lead-out region while multimode fiber (MMF) is the sensing region. The light emitted by the source is passed the lead-in region from the SMF and entered the MMF sensing region and experienced the diameter mismatch. The optical signal is more presented in the MMF rather than SMF. Hence, at the sensing region more signals are responded to the ambient environment. It is opposite phenomenon occurred on the MSM structure.
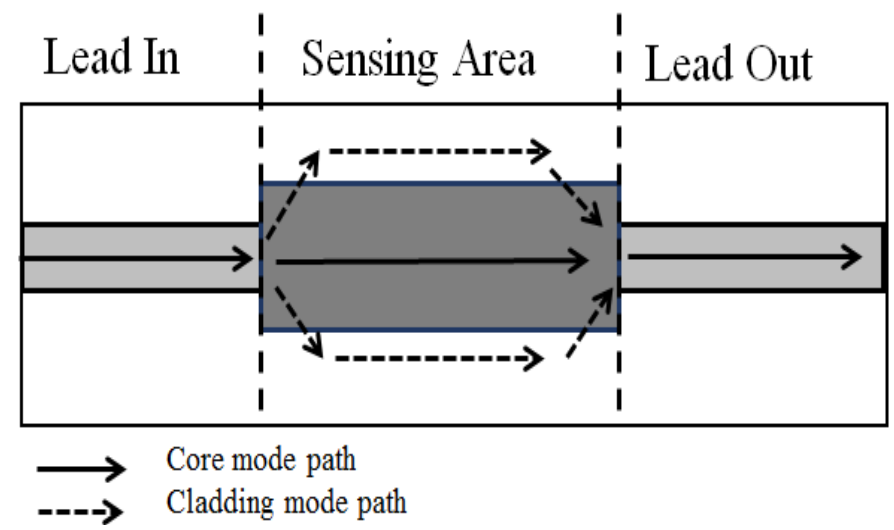

Figure 2 Block diagram of SMS structure.

Figure 3 shows the MSM structure in which the MMF acts as lead-in region and the lead-out region while SMF is the sensing-area region. The interference is occurred due to different core diameter of both SMF and MMF. The lead-in region through MSM structure is able to carry more optical signal compared to SMS structure.

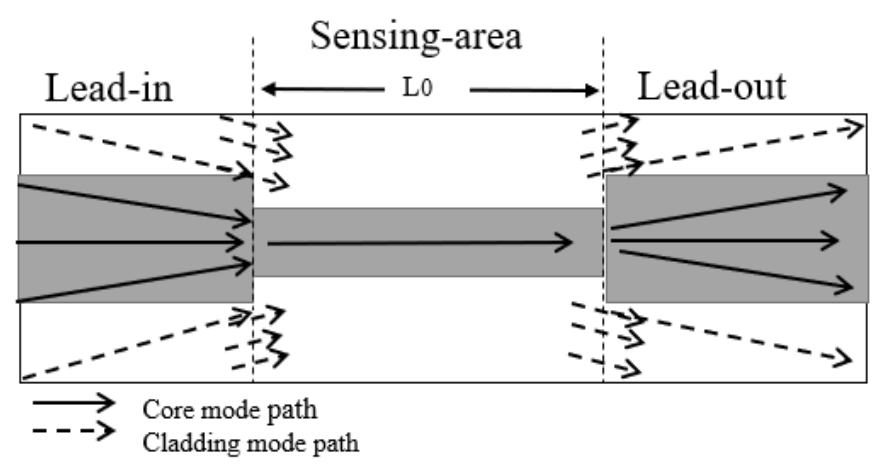

Figure 3 Block diagram of MSM structure.

When the optical signal is launched into sensing region through the lead-in region, multiple guided modes will be excited and propagated in between the lead-in region and sensing region [32]. Assuming the $m t h$ eigenmode of the lead-in region as $\Psi \mathrm{m}$, its excitation coefficient $b_{m}$ can be expressed as [33]

$$
b_{m}=\frac{\int_{0}^{\infty} E(r, 0) \Psi_{m}(r) r d r}{\int_{0}^{\infty} \Psi_{m}(r) \Psi_{m}(r) r d r}
$$

where $E(r, 0)$ is the field amplitude of the eigenmode transmitting to the splice point in between them and after propagating distance of the sensing region $L_{0}$ in the MMF in fields evolves into (2) where $\beta_{m}$ is the propagation constant of $m$ th eigenmode.

$$
E\left(r, L_{0}\right)=\sum_{m=1}^{M} b_{m} \Psi_{m}(r) \exp \left(j b_{m} L_{0}\right)
$$

The optical power of the core mode is partially coupled into the cladding modes of the sensing region due to the mode of the sensing SMF as $\Phi_{n}(r)(n=1$ for core mode, $n<1$ for cladding modes, then the excitation coefficient of each mode can be given by (3) and by estimating that all splices are ideal, only $\Phi_{L} P_{0 m}$ and $\Phi L P_{0 n}$ modes will be excited within the lead-in and sensing region due to the circular symmetry of the input field [33].

$$
c_{n}=\frac{\int_{0}^{\infty} E\left(r, L_{0}\right) \Phi_{n}(r) r d r}{\int_{0}^{\infty} \Phi_{n}(r) \Phi_{n}(r) r d r}
$$

A higher-order cladding mode employs a small proportion of the power, only the core mode and one cladding mode of the sensingregion are taken into consideration. The transmission spectrum of the interferometer is expressed as [7]:

$$
I=I_{1}+I_{2}+2 \sqrt{I_{1} I_{2}} \cos \left[\frac{2 \pi\left(n_{c o}^{e f f}-n_{c l}^{e f f}\right.}{\lambda} L_{0}\right]
$$

where $I_{1}$ and $I_{2}$ are the light intensities of the core mode and the cladding mode, $\lambda$ is the free-space optical wavelength in air, $L_{0}$ length of the sensing region and $n_{c o}^{e f f}, n_{c l}^{e f f}$ are indexes of the core and cladding:

$$
I=I_{\min } \text { when } \frac{2 \pi\left(n_{c o}^{e f f}-n_{c l}^{e f f}\right)}{\lambda} L_{0}=(2 k+1) \pi
$$

where $k$ is a positive integer. The wavelength of the interference is expressed as:

$$
\lambda=\frac{2 \pi\left(n_{c o}^{e f f}-n_{c l}^{e f f}\right)}{2 k+1} L_{0}
$$

Therefore, the fiber sensor will be affected by manipulating the length of sensing region [7]. The phase difference $(\Delta \emptyset)$ in between both regions changes linearly with wavelength: 


$$
\Delta \emptyset=\left(\beta_{m}-\beta_{m+1}\right) L_{0}=\frac{2 \sqrt{2 \Delta_{m}}}{a_{m}} L_{0}+\frac{4(m+1) \Delta_{m}}{\pi a_{m} n_{c o}} L_{0} \lambda
$$

where $n_{c o}, n_{c l}$ are the core and cladding indices, $a_{m}$ and $\Delta_{m}=\frac{n_{c o}^{2}-n_{c l}^{2}}{2 n_{c o}^{2}}$ are the core radius and the relative core-cladding refractive index difference and $L_{0}$ length of the sensing region. Due to the difference of refractive index and diameter size between the core and cladding, the core mode and the cladding modes will interfere in the sensingregion [16]. The effective index difference between the core and cladding modes of MMF caused the phase difference and the interference can be described as:

$$
\phi^{m}=\frac{2 \pi\left(n_{\text {eff }}^{\text {core }}-n_{\text {eff }}^{\text {cladding, } m}\right.}{\lambda} L_{0}=\frac{2 \pi \Delta n_{\text {eff }}^{m}}{\lambda} L_{0}
$$

where $\lambda, n_{\text {eff }}^{\text {core }}, n_{\text {eff }}^{\text {cladding, } m}, L_{0}, \Delta n_{\text {eff }}^{m}$ are the free space wavelength, the effective indexes of core mode and $m t h$ cladding mode, the length of the sensing region and the effective RI difference respectively [27] Then, a part of the core mode and cladding modes will be recoupled into the fundamental mode of the lead-out [28]. Space wavelength, the effective indexes of core mode and $m$ th cladding mode, the length of the sensing region, the effective refractive index difference respectively [27].

\section{MZI STRUCTURES DESIGN}

The proposed design of MZI structures would utilize the simple arc fusion splicing technique only. The fabrication technique employed the Fujikura FSM-18R as the splicer and a high-quality cleaver Fujikura CT-30 for flat and clean end faces uncoated fiber. An accurate measurement for each region was needed before cutting the SMF and MMF especially the length of the sensing region. The buffer jacket of sensing region of both structures were removed in order to expose the bare fiber directly to the surrounding. The fiber was cleaned with alcohol before being spliced. Details about the structures design have been recorded in Table 1 .

Table 1: Structures design parameter.

\begin{tabular}{lcc}
\hline \multicolumn{1}{c}{ Structures } & SMS & MSM \\
\hline Lead-in region and Lead- & SMF & MMF \\
out region & $8.2 / 125 \mu \mathrm{m}$ & $50 / 125 \mu \mathrm{m}$ \\
Size core/cladding & $1.4712 / 1.4959$ & $1.4849 / 1.5000$ \\
Refractive index & $\mathrm{MMF}$ & $\mathrm{SMF}$ \\
core/cladding & $25 / 125 \mu \mathrm{m}$ & $25 / 125 \mu \mathrm{m}$ \\
Sensing-area region & $4 \mathrm{~cm}, 8 \mathrm{~cm}$ & $4 \mathrm{~cm}, 8 \mathrm{~cm}$ \\
Size core/cladding & $1.4849 / 1.5000$ & $1.4712 / 1.4959$ \\
Length of sensing-area & \multicolumn{2}{c}{ Alcohol } \\
Refractive index & \multicolumn{2}{c}{} \\
core/cladding & \multicolumn{2}{c}{} \\
Cleaning agent & &
\end{tabular}

The SMS structure was fabricated by splicing a section of MMF in between two SMFs as shown in

Figure 2 while MSM structure spliced the SMF in between the MMFs as shown in

Figure 3. Both structures were spliced by a multimode auto mode for all regions.
Figure 4 (a) shows an aligned fibers, ready to be spliced. After applying arc discharge, the fiber tips were softened and pushed together and became a perfect one line fiber as

Figure 4 (b) with acceptable power loss of $2 \mathrm{~dB}$.

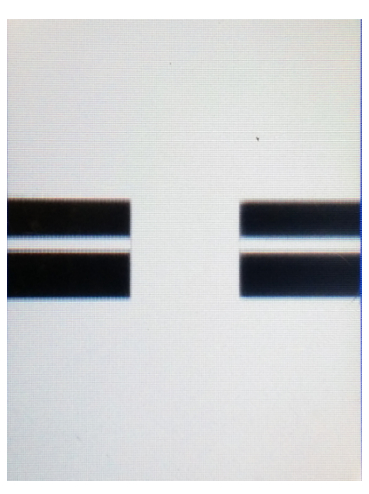

(a)

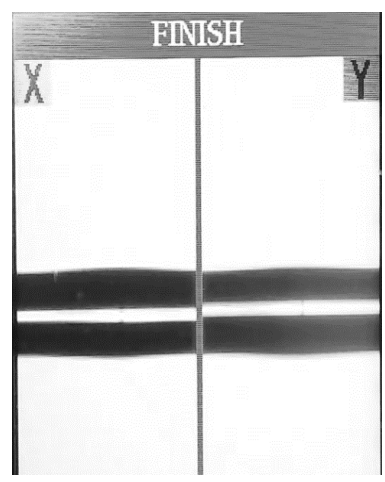

(b)
Figure 4 (a) Both fibers were aligned (b) Both fibers were successfully spliced.

\section{METHODOLOGY}

The experimental setup was arranged as presented in

Figure 5 to measure the changes of the operating wavelength. Details related to the experimental set-up have been recorded in Table 2. The lead-in region was connected to the optical source while the lead-out region was connected to the optical spectrum analyzer (OSA). The buffer jacket of the sensing-region has been removed and made it sensitive to the surrounding changes. The sensing region has been immersed in the various solutions and the readings were recorded. Both structures were exposed to the air of 1.000 RIU and became the reference operating wavelength. In order to obtain the standard deviation error, the readings were repeated five times. The sensor region was cleaned with acetone liquid and dried with air prior to another liquid testing.

Table 2 Sensor experimental set-up.

\begin{tabular}{ll}
\hline Specification & Description \\
\hline Type of optical source & Visual fault locator DXL \\
(650nm,10mW) \\
Type of OSA & Anritsu MS9740A \\
Type of refractometer & Digital SG/Brix Refractometer \\
Type of solutions and the & Water: 1.333 \\
refractive index. & 1 mol sucrose: 1.384 \\
Temperature & Oil :1.464 \\
Cleaning agent & Room temperature $27^{\circ} \mathrm{C}$ \\
\hline
\end{tabular}

The fabricated sensors were immersed in different solutions of different refractive indexes. Surrounding liquid affected the modal field distribution and mode propagation constants in the sensingregion through the evanescent wave, changing the phase condition of interference to move the output spectrum [34] as explained in (7). 


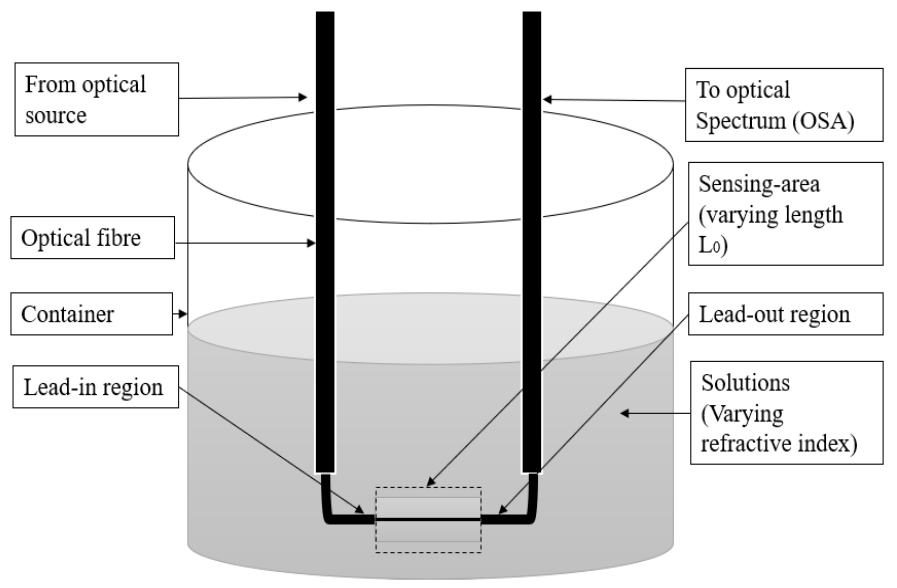

Figure 5 Experimental set-up.

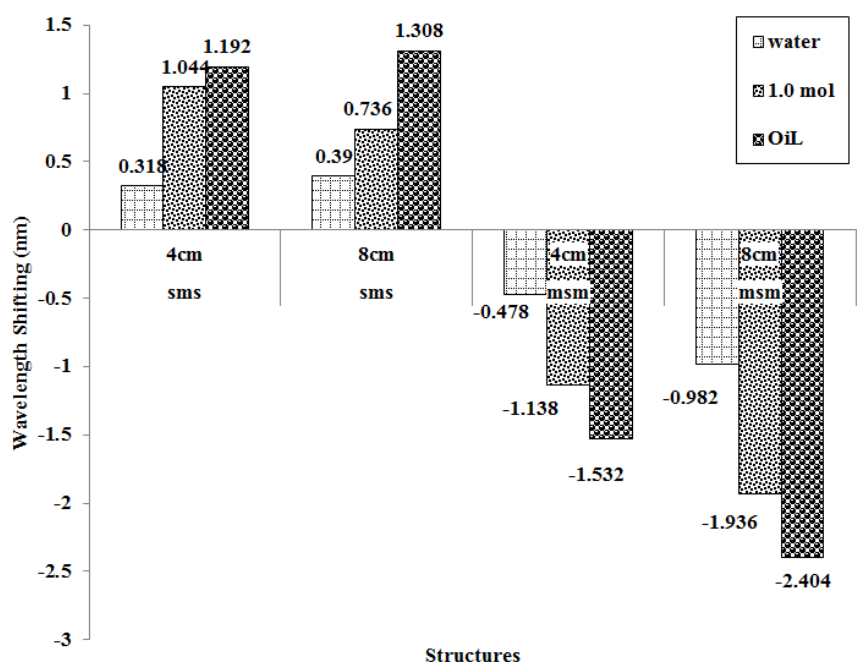

Figure 7 Wavelength shift of SMS and MSM structures.

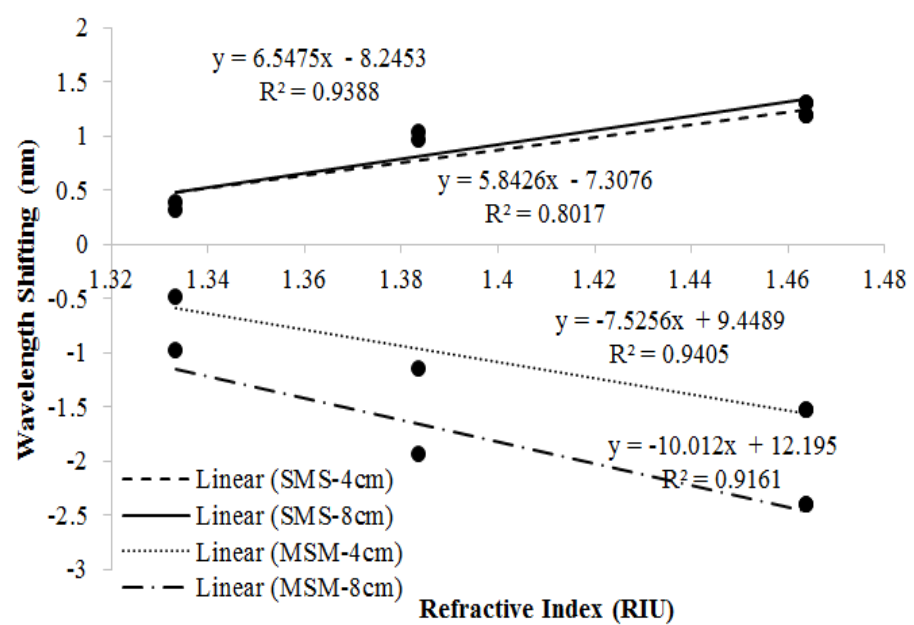

Figure 8 Linear fitting regression of SMS and MSM structures.

Figure 8 depicts the linear fitting regression graph for the sensitivity of both structure resulted from the wavelength shift response for different RIU and the length of the sensing-area. From the graph, the sensitivity values for SMS structure were

$5.8426 \mathrm{~nm} / \mathrm{RIU}$ and $6.5475 \mathrm{~nm} / \mathrm{RIU}$ while for MSM structure were $7.5256 \mathrm{~nm} /$ RIU and $-10.0120 \mathrm{~nm} /$ RIU for $4 \mathrm{~cm}$ and $8 \mathrm{~cm}$ of sensor region length respectively. It was observed that the sensor region with $8 \mathrm{~cm}$ length demonstrated a higher sensitivity for each structure. It could be concluded that the MSM structure demonstrated greater sensitivity compared to the SMS structure.

\section{CONCLUSION}

In summary, a compact MZI sensor devices were demonstrated based on SMS and MSM structure. The performance of sensor device was investigated on different types of refractive index corresponding to different lengths of the sensing region of $4 \mathrm{~cm}$ and $8 \mathrm{~cm}$. Experimental results showed that the operating wavelength of the sensor device experienced a redshift and blueshift as the value of refractive index was increased for both SMS and MSM respectively. Another crucial observation was the length of the sensing region influenced the sensitivity of the sensor device. The sensitivity of $8 \mathrm{~cm}$ length for both structures was more sensitive with $6.5475 \mathrm{~nm} / \mathrm{RIU}$ for SMS structure and $-10.0120 \mathrm{~nm} / \mathrm{RIU}$ for MSM structure. In could be deduced that the MSM was more sensitive than the SMS structure under the same platform of this work. The findings should make an length recorded more wavelength shift compared to the $4 \mathrm{~cm}$ sensor's length corresponding to every tested solution. 
important reference in designing an improved fiber optic sensor by manipulating parameters and selection of MZI structures.

\section{ACKNOWLEDGEMENT}

The authors would like to thank Centre for Telecommunication Research \& Innovation (CeTRI) and Universiti Teknikal Malaysia Melaka (UTeM) for the financial support.

\section{REFERENCES}

[1] Y. Dai, "Highly sensitive liquid-level sensor based on weak uniform fiber Bragg grating with narrow-bandwidth," Opt. Eng., vol. 51, no. 4, pp. 44401, 2012.

[2] X. Hao, Z. Tong, W. Zhang, and Y. Cao, "A Fiber Laser Temperature Sensor based on SMF Core-offset Structure," Opt. Commun., vol. 335, pp. 78-81, 2015 .

[3] S. Yuan, Z. Tong, J. Zhao, W. Zhang, and Y. Cao, "High temperature fiber sensor based on spherical-shape structures with high sensitivity," Opt. Commun., vol. 332, pp. 154-157, 2014

[4] R. Guyard, D. Leduc, Y. Lecieux, and C. Lupi, "Superposition of fiber Bragg and LPG gratings for embedded strain measurement," Comptes Rendus Physique, vol. 17, no. 9, pp. 1027-1037, 2016.

[5] I.-L. Bundalo, R. Lwin, S. Leon-Saval, and A. Argyros, "All-plastic fiber-based pressure sensor," Appl. Opt., vol. 55, no. 4, p. 811, 2016.

[6] S. Azad, E. Sadeghi, R. Parvizi, A. Mazaheri, and M. Yousefi, "Sensitivity optimization of $\mathrm{ZnO}$ clad-modified optical fiber humidity sensor by means of tuning the optical fiber waist diameter," Opt. Laser Technol., vol. 90, no. September 2016, pp. 96-101, 2017.

[7] L. Cai, Y. Zhao, and X.-G. Li, "Applications of Modal Interferences in Optical Fiber Sensors Based on Mismatch Methods," Instrum. Sci. Technol., vol. 43, no. 1, pp. 1-20, 2014.

[8] G. An et al., "Extra-broad Photonic Crystal Fiber Refractive Index Sensor Based on Surface Plasmon Resonance," Plasmonics, vol. 12, no. 2, pp. 465-471, 2017.

[9] Q. Wang, C. Li, C. Zhao, and W. Li, "Guided-mode-leaky-mode-guidedmode fiber interferometer and its high sensitivity refractive index sensing technology," Sensors (Switzerland), vol. 16, no. 6, pp. 1-11, 2016.

[10] A. D. Gomes and O. Frazão, "Mach-Zehnder Based on Large Knot Fiber Resonator for Refractive Index Measurement," IEEE Photonics Technol. Lett., vol. 28, no. 12, pp. 1279-1281, 2016.

[11] Y. Ma et al., "Mach-Zehnder interferometer based on a sandwich fiber structure for refractive index measurement," IEEE Sens. J., vol. 12, no. 6, pp. 2081-2085, 2012.

[12] T. K. Yadav, R. Narayanaswamy, M. H. Abu Bakar, Y. M. Kamil, and M. a. Mahdi, "Single mode tapered fiber-optic interferometer based refractive index sensor and its application to protein sensing," Opt. Express, vol. 22, no. 19, pp. 22802, 2014.

[13] H. Tazawa, T. Kanie, M. Katayama, "Fiber-optic coupler based refractive index sensor and its application to biosensing," Appl. Phys. Lett., vol. 91 no. 11 , pp. 10-13, 2007.

[14] A. K. Mishra, S. K. Mishra, A. P. Singh, "Giant infrared sensitivity of surface plasmon resonance-based refractive index sensor," Plasmonics, pp. 1-8, 2017.

[15] H. Wei, Y. Zhu, S. Krishnaswamy, "Optofluidic Photonic crystal fiber coupler for measuring the refractive index of liquids," IEEE Photonics Technol. Lett., vol. 28, no. 1, pp. 103-106, 2016.

[16] R. K. Gangwar V. K. Singh, "Highly Sensitive surface plasmon resonance based d-shaped photonic crystal fiber refractive index sensor," Plasmonics, pp. 1-6, 2016.

[17] B. H. Lee, Y. H. Kim, K. S. Park, J. B. Eom, M. J. Kim, B. S. Rho, H. Y. Choi, "Interferometric fiber optic sensors," Sensors, vol. 12, no. 3, pp.
2467-2486, 2012

[18] E. Vargas-Rodriguez, A. D. Guzman-Chavez, M. Cano-Contreras, E. Gallegos-Arellano, D. Jauregui-Vazquez, J. C. Hernández-García, J. M. Estudillo-Ayala, R. Rojas-Laguna, "Analytical modelling of a refractive index sensor based on an intrinsic micro Fabry-Perot interferometer," Sensors (Basel)., vol. 15, no. 10, pp. 26128-26142, 2015.

[19] D. Jáuregui-Vázquez, J. M. Estudillo-Ayala, R. Rojas-Laguna, E. Vargas-Rodríguez, J. M. Sierra-Hernández, J. C. Hernández-García, R. I. Mata-Chávez, "An all fiber intrinsic Fabry-Perot interferometer based on an air-microcavity," Sensors (Switzerland), vol. 13, no. 5, pp. 63556364, 2013.

[20] D. Wu, T. Zhu, Guo-Yin Wang, Jian-Yu Fu, X.-G. Lin, and G.-L. Gou, "Intrinsic fiber-optic Fabry-Perot interferometer based on arc discharge and single-mode fiber.," Appl. Opt., vol. 52, no. 12, pp. 2670-2675, 2013.

[21] J. Zhou, Y. Wang, C. Liao, B. Sun, J. He, G. Yin, S. Liu, G. Wang, X. Zhong, J. Zhao, "Intensity modulated refractive index sensor based on optical fiber Michelson interferometer," Sensors Actuators, B Chem., vol. 208, pp. 315-319, 2015.

[22] S. Zhang, T. Yuan, L. Yuan, "Asymmetrical twin-core fiber based Michelson interferometer for environmental refractive index sensing," in Proc. SPIE (25th Int. Conference on Optical Fiber Sensors), vol. 10323, 1032377, 2017.

[23] Y. C. Liang, C. C. Liao, Y. L. Lo, "Abrupt taper Michelson interferometer using heterodyne for measuring refractive index," IEEE Photonics Technol. Lett., vol. 26, no. 23, pp. 2330-2333, 2014.

[24] J. Kang, X. Dong, C. Zhao, and Y. Zhao, "A sagnac loop sensor for refractive index measurement," 2011 Symp. Photonics Optoelectron. SOPO 2011, pp. 0-3, 2011.

[25] X. hu Fu, K. li Yang, J. peng Zhang, Y. hua Dong, "A tension insensitive $\mathrm{PbS}$ fiber temperature sensor based on Sagnac interferometer," Optoelectron. Lett., vol. 13, no. 2, pp. 135-137, 2017.

[26] T. Schubert, N. Haase, and H. Ktick, "Refractive-index measurements using an integrated Mach-Zehnder interferorneter," Sensors and Actuators, vol. 60, no. 100, pp. 108-112, 1997.

[27] X. Sun, X. Dong, Y. Hu, H. Li, D. Chu, J. Zhou, C. Wang, J. Duan, “A robust high refractive index sensitivity fiber Mach - Zehnder interferometer fabricated by femtosecond laser machining and chemical etching," Sensors Actuators A. Phys., vol. 230, pp. 111-116, 2015.

[28] H. Wang et al., "Simultaneous measurement of refractive index and temperature based on asymmetric structures modal interference," Opt. Commun., vol. 364, pp. 191-194, 2016.

[29] J. Harris, P. Lu, H. Larocque, L. Chen, and X. Bao, "In-fiber MachZehnder interferometric refractive index sensors with guided and leaky modes," Sensors Actuators, B Chem., vol. 206, pp. 246-251, 2015.

[30] H. Sun, J. Zhang, S. Yang, Q. Rong, "Temperature and refractive index sensing characteristics of an MZI-based multimode fiber-dispersion compensation fiber-multimode fiber structure," Opt. Fiber Technol., vol. 18, no. 6, pp. 425-429, 2012.

[31] J. Wo, G. Wang, Y. Cui, Q. Sun, R. Liang, P. P. Shum, D. Liu, "Refractive index sensor using microfiber-based Mach-Zehnder interferometer," Opt. Lett., vol. 37, no. 1, p. 67, 2012.

[32] Z. Xu, Q. Sun, J. Wo, Y. Dai, X. Li, and D. Liu, "Volume strain sensor based on spectra analysis of in-fiber modal interferometer," IEEE Sens. $J$. , vol. 13, no. 6, pp. 2139-2145, 2013.

[33] Q. Wu, Y. Semenova, B. Yan, Y. Ma, P. Wang, C. Yu, G. Farrell, "Fiber refractometer based on a fiber Bragg grating and single-modemultimode-single-mode fiber structure," Opt. Lett., vol. 36, no. 12, pp. 2197-2199, 2011.

[34] Y. Zhao, L. Cai, X. G. Li, and F. C. Meng, "Liquid concentration measurement based on SMS fiber sensor with temperature compensation using an FBG," Sensors Actuators, B Chem., vol. 196, pp. 518-524, 2014. 